\title{
WEIGHTED INEQUALITIES FOR COMMUTATORS OF FRACTIONAL AND SINGULAR INTEGRALS
}

\author{
Chrlos Segovia and José L. Torrea
}

\section{Introduction}

We dedicate this paper to the memory of José Luis Rubio de Francia, who developed the theory of extrapolation and gave beautiful applications of vectorial methods in harmonic analysis.

Through this paper we shall work on $\mathrm{R}^{n}$, endowed with the Lebesguc measure. Given a Banach space $E$ we shall denote by $L_{E}^{p}\left(\mathbf{R}^{n}\right)$ or $L_{E}^{p}$ the BochnerLebesgue space of $\mathrm{E}$-valucd strongly measurable functions such that

$$
\int\|f(x)\|_{E}^{p} d x<+\infty
$$

Given a positive measurable function $\alpha(x)$ we shall denote by $L_{E}^{p}(\alpha)$ the space of E-valued strongly measurable functions such that $\int\|f(x)\|_{E}^{p} \alpha(x) d x<\infty$ and we shall denote by $B M O_{E}(\alpha)$ the space of strongly measurable functions $b$ such that

$$
\int_{Q}\left\|b(x)-b_{Q}\right\|_{E} d x \leq C \int_{Q} \alpha(x) d x
$$

where

$$
b_{Q}=|Q|^{-1} \int_{Q} b(x) d x .
$$

Given two Banach spaces $E$ and $F$, we shall denote by $\mathcal{L}(E, F)$ the Banach space of all continuous linear operators from $E$ into $F$.

By a Banach lattice we mean a partially ordered Banach space $F$ over the reals such that

(i) $x \leq y$ implies $x+z \leq y+z$ for every $x, y, z \in F$,

(ii) $a x \geq 0$ for every $x \geq 0$ in $F$ and $a \geq 0$ in $\mathbf{R}$.

(iii) for cvery $x, y \in F$ there exists a least upper bound (l.u.b.) and a greatest lower bound (g.l.b.), and

(iv) if $|x|$ is defined as $|x|=\mid$.u.b. $(x,-x)$ then $\|x\| \leq\|y\|$ whenever $|x| \leq|y|$. 
We shall say that a positive function $\alpha$ belongs to $A(p, q)$ if

$$
\left(\frac{1}{|Q|} \int_{Q} \alpha^{-p^{\prime}}(x) d x\right)^{1 / p^{\prime}}\left(\frac{1}{|Q|} \int_{Q} \alpha^{q}(x) d x\right)^{1 / g} \leq C,
$$

holds for any cube $Q \subset \mathrm{R}^{n}$ and $p^{3}+p=p^{p} p$, the constant $C$ not depending on $Q$.

Observe that if we denote by $A_{p}$ the Muckenhoupt's class, then, for $p>1$, $\omega \in A(p, p)$ if and only if $\omega^{p} \in A_{p}$.

Finally we shall say that a Banach space E is U.M.D. if the Hilbert transform is bounded from $L_{E}^{2}$ into $L_{E}^{2}$, see [2].

The paper is organized as follows: in section 1 we state and prove the extrapolation results, in section 2 we state the commutator theorems, thcse theorems are proved in section 4, we give several applications in section 3 .

\section{Two extrapolation results}

Let $\nu \geq 0$ be a measurable function, $1<p \leq q<\infty, 1<\lambda \leq \infty$ and $\frac{1}{p}-\frac{1}{q}=\frac{1}{\lambda}$. We shall say that a weight $\omega$ belongs to the class $A^{(v)}(p, q)$ if

$$
\omega \in A(p, q) \text { and } \nu \omega \in A(p, q) \text {. }
$$

Let $p \geq 1$, we shall say that $\omega$ belongs to the class $A_{p}^{(\nu)}$ if $\omega \in A_{p}$ and $\nu^{\nu_{\omega}} \in A_{p}$.

Observe that $1=\lambda^{\prime}\left(\frac{1}{p^{\prime}}+\frac{1}{q}\right)$, then it is clear that $\omega \in A^{(\nu)}(p, q)$ if and only if $\omega^{-p^{\prime}} \in A_{1+p^{\prime} / q}^{\left(\nu^{-\lambda^{\prime}}\right)}$ and if and only if $\omega^{q} \in A_{1+q / p^{\prime}}^{\left(\nu^{\lambda^{\prime}}\right)}$ therefore by the properties of the class $A_{r}^{(\nu)}$, see [7], the class $A^{(\nu)}(p, q)$ is not empty if and only if $\nu^{\lambda^{\prime}} \in A_{2}$.

We shall use the following lemma, due to Rubio de Francia for the classes $A_{p}$, whose proof for the classes $A_{p}^{(\nu)}$ can be found in [ 7 ].

(1.I) Lemma. Assume $\nu \in A_{2}$, let $1<r<\infty$ and $\omega \in A_{r}^{(\nu)}$. Then, for uniy positive: $u$ with $u \in L^{r^{\prime}}(\omega)$ there exists $U \in L^{r^{\prime}}(\omega)$ such that

(a) $* \leq U$ a.e.

(li) $\|U\|_{l r^{\prime}(w)} \leq C\|u\|_{l, r^{\prime}(\omega)}$ and

(c) $U \omega \in A_{1}^{(\nu)}$.

Now wo: stizts: the: matin testults of this paragraph.

(1.2) Theorem. Let $T$ bre a sublinear operator defined on $C_{0}^{\infty}$ and satisfying

$$
\|\omega T f\|_{\infty} \leq C_{\omega}\|\omega f\|_{\infty},
$$


for every $\omega$ such that $\omega^{-1} \in A_{1}$ and $(\nu \omega)^{-1} \in A_{1}$. Then

$$
\|T f\|_{L^{p}(\omega)} \leq C_{\psi}\|f\|_{L p(\omega)}
$$

holds for every $\omega \in A_{p}^{(\nu)}$ and $p>1$.

(1.3) Theorem. Let $1<\lambda \leq \infty$ and $T$ be a sublinear operator defined on $C_{0}^{\infty}$ and satisfying

$$
\|\omega T f\|_{\infty} \leq C_{\omega}\|f\|_{L^{\lambda}\left(\omega^{\lambda}\right)},
$$

for every $\omega$ such that $\omega^{-\lambda^{\prime}} \in A_{1}$ and $(\nu \omega)^{-\lambda^{\prime}} \in A_{1}$. Then if $1<p<\lambda, \frac{1}{p}-\frac{1}{q}=$ $\frac{1}{\lambda}$ the inequality

$$
\|T f\|_{L^{Q}(\omega \eta)} \leq C_{\omega} \|\left. f\right|_{L^{p}\left(\omega^{p}\right)}
$$

holds for every $\omega \in A^{(\nu)}(p, q)$.

The proof of Theorem (1.2) can be found in [7], we shall reproduce it here for the sake of completeness.

Lct $f \in L^{p}(\omega), \omega \in A_{p}^{(v)}, 1<p$. We define

$$
g=\omega^{1 / p(p-1)}|f| \omega^{1 / p} /\left(\int|f|^{p} \omega\right)^{1 / p} \quad \text { if } \quad|f| \omega^{1 / p} \neq 0
$$

and

$$
g=\omega^{1 / p(p-1)} e^{-\pi|x|^{2} / p} \quad \text { if } \quad|f| \omega^{1 / p}=0 .
$$

Then, $g>0$ a.e., $\int g^{p} \omega^{-p^{\prime} / p} \leq 2$, and

$$
\left\|f \omega^{p / p} g^{-1}\right\|_{\infty}=\left(\int|f|^{p} \omega d x\right)^{1 / p} .
$$

Now, by the properties of the classes $A_{r}^{(v)}$, see [7], $\omega^{-p^{\prime} / p} \in A_{p}^{(\nu)}$, therefore we can apply lemma (1.1) and we obtain a function $G \geq g$ a.e., $G \omega^{1-p^{\prime}} \in A_{p^{\prime}}^{(\nu)}$, and satisfying

$$
\int G^{p} \omega^{1-p^{\prime}} d x \leq c \int g^{p} \omega^{1-p^{t}} d x \leq 2 c .
$$

Then,

$$
\left(\int|f|^{p} \omega d x\right)^{1 / p} \geq C\left\|\omega^{p^{\prime}-1} G^{-1} f\right\|_{\infty}
$$

Since $\left(\omega^{p^{t}-1} G^{-1}\right)^{-1}$ and $\left(\nu \omega^{p^{\prime}-1} G^{-1}\right)^{-1}$ belong to $A_{1}$, we get

$$
\begin{aligned}
\left(\int|f|^{p} \omega d x\right)^{3 / p} & \geq c\left\|\omega^{p^{\prime}-1} G^{-1} T f\right\|_{\infty} \\
& \geq c^{t}\left\|\omega^{p^{\prime}-1} G^{-1} T f\right\|_{\infty}\left(\int G^{p} \omega^{1-p^{\prime}} d x\right)^{1 / p} \\
& \geq c^{\prime}\left(\int|T f|^{p} \omega d x\right)^{1 / p}
\end{aligned}
$$


as we wanted to show. Since

Proof of Theorem (1.3): Let $\omega \in A^{(\nu)}(p, q), \frac{1}{p}-\frac{1}{q}=\frac{1}{\lambda}$ and $f \in L^{p}\left(\omega^{\nu}\right)$.

$$
\left(\int|f|^{p} \omega^{p} d x\right)^{3 / p}=\left(\int\left(\left|f \omega^{p^{\prime}}\right|^{\lambda}\right)^{p / \lambda} \omega^{-p^{\prime}} d x\right)^{(\lambda / p)(1 / \lambda)},
$$

there exists $g \geq 0$ such that

$$
\int g^{(p / \lambda)^{\prime}} \omega^{-p^{\prime}} d x=1
$$

and

$$
\left(\int|f|^{p} \omega^{p} d x\right)^{1 / p}=\left(\int\left|f \omega^{p^{\prime}}\right|^{\lambda} g \omega^{-p^{\prime}} d x\right)^{1 / \lambda}
$$

Let $h=g^{\lambda^{\prime} / \lambda}$. Then (1.4) is equivalent to

$$
\int h^{q / \lambda^{\prime}} \omega^{-p^{\prime}} d x=1
$$

Since $\omega \in A^{(\nu)}(p, q)$ we have $\omega^{-p^{\prime}} \in A_{1+p^{\prime} / q}^{\left(\nu^{-\lambda^{\prime}}\right)}$; setting $r=1+\frac{p^{\prime}}{q}$, we can apply lemma (1.1), observing that $r^{\prime}=\frac{q}{\lambda^{\prime}}$, to obtain a function $H \geq h$ such that

$$
\int H^{q / \lambda^{\prime}} \omega^{-p^{\prime}} d x \leq c \quad \text { and } \quad H \omega^{-p^{\prime}} \in A_{1}^{\left(\nu^{-\lambda^{\prime}}\right)} .
$$

Therefore the weight $v=H^{-1 / \lambda^{\prime}} \omega^{p^{\prime} / \lambda^{\prime}}$ is such that $v^{-\lambda^{\prime}} \in A_{1}$ and $(\nu v)^{-\lambda^{\prime}} \in$ $A_{1}$.

Then, returning to (1.5) and using the hypothesis we have

$$
\begin{aligned}
& \left(\int|f|^{p} \omega^{p} d x\right)^{1 / p} \geq\left(\int|f|^{\lambda}\left(h^{-1 / \lambda^{\prime}} \omega^{p^{\prime} / \lambda^{\prime}}\right)^{\lambda} d x\right)^{1 / \lambda} \\
& \geq\left(\int|f|^{\lambda}\left(H^{-1 / \lambda^{\prime}} \omega^{p^{\prime} / \lambda^{\prime}}\right)^{\lambda} d x\right)^{1 / \lambda} \geq c\left\|(T f) H^{-1 / \lambda^{\prime}} \omega^{p^{\prime} / \lambda^{\prime}}\right\|_{\infty} .
\end{aligned}
$$

Taking (1.6) into account, this is bigger than

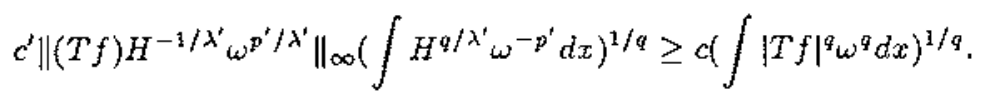

Note. The theorems of this section are heavily inspired in [10]. 


\section{Commutators for fractional and singular integrals}

(2.1) Definition: we shall derote by $B M O_{E}(\alpha)$ the space of strongly measurable functions $b$ such that

$$
\int_{Q}\left\|b(x)-b_{Q}\right\|_{E} d x \leq C \int_{Q} \alpha(x) d x
$$

where

$$
b_{Q}=|Q|^{-1} \int_{Q} b(x) d x
$$

(2.2) Definition: We shall say that a positive function $\alpha$ belongs to $A(p, q)$ if

$$
\left(\frac{1}{|Q|} \int_{Q} \alpha^{-p^{\prime}}(x) d x\right)^{1 / p^{\prime}}\left(\frac{1}{|Q|} \int_{Q} \alpha^{q}(x) d x\right)^{1 / q} \leq C
$$

holds for any cube $Q \subset \mathrm{R}^{n}$ and $p^{\prime}+p=p^{\prime} p$, the constant $C$ not depending on $Q$.

Now we state the theorems of this section.

(2.3) Theorem. Let $E, F$ be Banach spaces. Let $T$ be a bounded linear operator from $L_{E}^{p}\left(\mathbf{R}^{n}\right)$ into $L_{F}^{q}\left(\mathbf{R}^{n}\right)$ for $1<p \leq q<\infty, 0 \leq \gamma<n$ and $\frac{1}{p}-\frac{1}{q}=\frac{\gamma}{n}$. Assume that there exists an $\mathcal{L}(E, F)$-valued kernel satisfying:

(K.1) for any compactly supported $f$,

$$
T f(x)=\int k(x, y) f(y) d y, \quad \text { for } \quad x \notin \operatorname{supp} f,
$$

(K.2) if $|x-y|>2\left|x-x^{\prime}\right|$ then

$$
\left\|k(x, y)-k\left(x^{\prime}, y\right)\right\| \leq \frac{C\left|x-x^{\prime}\right|}{|x-y|^{n+1-\gamma}},
$$

Let $\ell \rightarrow \tilde{\ell}$ be a bounded linear operator from $\mathcal{L}(E, E)$ into $\mathcal{L}(F, F)$ such that

$$
\begin{gathered}
\tilde{\ell} T f(x)=T(\ell f)(x) \quad \text { and } \\
k(x, y) \ell=\tilde{\ell} k(x, y) .
\end{gathered}
$$

(2.4) Given $\alpha, \beta \in A(p, q), \nu=\alpha \beta^{-1}$ and $b$ an $\mathcal{L}(E, E)$-valued function such that $b \in B M O_{\mathcal{L}(E, E)}(\nu)$ and $\tilde{b} \in B M O_{\mathcal{L}(F, F)}(\nu)$, then, the operator $C_{b}$ defined by

$$
C_{b} f(x)=\tilde{b}(x) T f(x)-T(b f)(x),
$$

is bounded from $L_{E}^{p}\left(\alpha^{p}\right)$ into $L_{F}^{q}\left(\beta^{q}\right)$ for $1<p \leq q<\infty$ and $\frac{1}{p}-\frac{1}{q}=\frac{\gamma}{n}$. 
(2.5) Given $\alpha, \beta \in A(p, q), \mu^{2}=\alpha \beta^{-1}$, a and $b \mathcal{L}(E, E)$-valued functions such that $a, b \in B M O_{\mathcal{C}(E, E)}(\mu)$ and $\tilde{a}, \tilde{b} \in B M O_{\mathcal{L}(F, F)}(\mu)$, moreover, for every $x$ and $y, a(x) b(y)=b(y) x(a)$ and $\tilde{a}(x) \tilde{b}(x)=\tilde{b}(x) \tilde{a}(x)$. Then, the operator $C_{a, b}$ defined by

$$
C_{a, b} f(x)=\tilde{b}(x) C_{a} f(x)-C_{a}(b f)(x),
$$

is bounded from $L_{E}^{p}\left(\alpha^{p}\right)$ into $L_{F}^{q}\left(\beta^{q}\right)$ for $1<p \leq q<\infty$ and $\frac{1}{p}-\frac{1}{q}=\frac{\gamma}{n}$.

(2.6) Theorem. Let $F$ be a Banach lattice and $V$ a bounded linear operator from $L^{p}\left(\mathbf{R}^{n}\right)$ into $L_{F}^{q}\left(\mathbf{R}^{n}\right)$ for $1<p \leq q<\infty, 0 \leq \gamma<n$ and $\frac{1}{p}-\frac{1}{q}=\frac{\gamma}{n}$. Assume that there exists an $F$-valued kernel $W(x, y)$ satisfying

(W.1) $W(x, y)$ is positive for every $x$ and $y$,

(W.2) for any $f$ with compact support

$$
V f(x)=\int W(x, y) f(y) d y, \text { and }
$$

(W.3) if $|x-y|>2\left|x-x^{\prime}\right|$, then

$$
\left\|W(x, y)-W\left(x^{\prime}, y\right)\right\| \leq \frac{C\left|x-x^{\prime}\right|}{|x-y|^{n+1-\gamma}} .
$$

(2.7) Given $\alpha, \beta \in A(p, q), \nu=\alpha \beta^{-1}$ and $b \in B M O(\nu)$, then the operator $V_{b}^{+}$defined by

$$
V_{b}^{+} f(x)=\int|b(x)-b(y)| W(x, y) f(y) d y
$$

is bounded from $L^{p}\left(\alpha^{p}\right)$ into $L_{F}^{q}\left(\beta^{q}\right)$ for $1<p \leq q<\infty$ and $\frac{1}{p}-\frac{1}{q}=\frac{\gamma}{n}$.

(2.8) Given $\alpha, \beta \in A(p, q), \mu^{2}=\alpha \beta^{-1}$, $a$ and $b$ functions in $B M O(\mu)$, then, the operator $V_{a, b}^{+}$defined by

$$
V_{a, b}^{+} f(x)=\int|a(x)-a(y)| b(x)-b(y) \mid W(x, y) f(y) d y,
$$

is bounded from $L^{p}\left(\alpha^{p}\right)$ into $L_{F}^{q}\left(\beta^{q}\right)$ for $1<p \leq q<\infty$ and $\frac{1}{p}-\frac{1}{q}=\frac{\gamma}{n}$.

(2.9) Remark. If $\nu^{2} \in A_{2}$ then $b \in B M O(\nu)$ if and only if

$$
\left(\frac{1}{|Q|} \int_{Q}\left|b(x)-b_{Q}\right|^{2} d x\right)^{1 / 2} \leq \frac{C \nu(Q)}{|Q|}
$$

To sec this it is enough to observe that if $\nu^{2} \in A_{2}$ then $\nu$ satisfies a reverse Holder condition with exponent 2, see [9]. 
(2.10) Remark. The theory of vector-valucd Calderon-Zygmund operators, see [5], and potential operators, see [6], can be applied in both theorems despite of the fact that smoothness is required only on the first variable of the kernel. Thus the operator $T$ (respectively $V$ ) turns out to be a bounded operator from $L_{E}^{p}\left(\alpha^{p}\right)$ into $L_{F}^{q}\left(\alpha^{q}\right)$ (respectively from $L^{p}\left(\alpha^{p}\right)$ into $\left.L_{F}^{q}\left(\alpha^{q}\right)\right)$ for $\alpha \in A(p, q)$, $\frac{1}{p}-\frac{1}{q}=\frac{\gamma}{n}$.

(2.11) Remark. Let $\nu^{2} \in A_{2}$, and $\alpha, \beta$ such that $\alpha \beta^{-1}=\nu^{2}$. It is easy to check that if $\delta=\alpha^{1 / 2} \beta^{1 / 2}$, then $\delta^{-1}$ belongs to $A_{1}$ if $\alpha^{-1}$ and $\beta^{-1}$ belongs to $A_{1}$ and $\delta \in A(p, q)$ if $\alpha$ and $\beta$ belong to $A(p, q)$.

\section{Applications}

A. Let $0 \leq \gamma<n$. Let $T$ be a bounded linear operator from $L^{p}\left(\mathbf{R}^{n}\right)$ into $L^{q}\left(\mathbf{R}^{n}\right)$ for $\frac{1}{p}-\frac{1}{q}=\frac{\gamma}{n}$.

Assume that there exists a kernel $k(x, y)$ that satisfies

(i) for any compactly supported $f$,

$$
T f(x)=\int k(x, y) f(y) d y \quad \text { if } \quad x \notin \text { supp } f, \quad \text { and }
$$

(ii) if $|x-y|>2\left|x-x^{i}\right|$, then

$$
\left|k(x, y)-k\left(x^{\prime}, y\right)\right| \leq C \frac{\left|x-x^{\prime}\right|}{|x-y|^{n+1-\gamma}} .
$$

Given $\frac{1}{p}-\frac{1}{q}=\frac{\gamma}{n}, a$ and $b$ in $B M O(\nu)$, we have, (3.1) for any pair $\alpha, \beta \in A(p, q), \nu=\alpha \beta^{-1}$, the commutator

$$
\left[T, M_{b}\right] f(x)=b(x) T f(x)-T(b f)(x)
$$

is bounded from $L^{p}\left(\alpha^{p}\right)$ into $L^{q}\left(\beta^{q}\right)$,

(3.2) for any pair $\alpha, \beta \in A(p, q), \nu^{2}=\alpha \beta^{-1}$, the commutator

$$
\left[\left[T, M_{b}\right], M_{a}\right] f(x)=a(x)\left[T, M_{b}\right] f(x)-\left[T, M_{b}\right](a f)(x)
$$

is bounded from $L^{p}\left(\alpha^{p}\right)$ into $L^{q}\left(\beta^{q}\right)$.

In particular, the commutator of any Calderón-Zygmund operator with standard kernel will be bounded from $L^{p}(\alpha)$ into $L^{p}(\beta)$ for $\alpha, \beta \in A_{p}$ and $\alpha \beta^{-1}=$ $\nu^{p}$. Also the commutator of the fractional integral of order $\gamma$ will be bounded from $L^{p}\left(\alpha^{p}\right)$ into $L^{q}\left(\beta^{q}\right), \frac{1}{p}-\frac{1}{q}=\frac{\gamma}{n}, \alpha, \beta \in A(p, q)$ and $\alpha \beta^{-1}=\nu$. Analogous restlts are true for the second commutator assuming $\nu=\mu^{2}$. For the case of the Hilbert transform see $[1]$, for the case of singular integrals with unbounded 
kernel see [7], and for the case of fractional integrals, see [3] for an unweighted version.

B. Let $T, k, \alpha, \beta, \nu, a$ and $b$ as in application $A$; and assume that in addition $k$ satisfies

$$
\begin{aligned}
& |k(x, y)| \leq \frac{c}{|x-y|^{n-\gamma}} \quad \text { and } \\
& \text { p.v. } \int k(x, y) f(y) d y \quad \text { exists a.e. }
\end{aligned}
$$

We define

$$
\begin{gathered}
T_{\varepsilon} f(x)=\int_{|x-y|>\varepsilon} k(x, y) f(y) d y, \\
C_{b}^{*} f(x)=\sup _{\varepsilon}\left|b(x) T_{\varepsilon} f(x)-T_{\varepsilon}(b f)(x)\right|
\end{gathered}
$$

and

$C_{a, b}^{*} f(x)=\sup _{\varepsilon}\left|a(x) b(x) T_{e} f(x)-a(x) T_{\varepsilon}(b f)(x)-b(x) T_{\varepsilon}(a f)(x)+T_{\varepsilon}(a b f)(x)\right|$.

Then

(3.3) for any pair $\alpha, \beta \in A(p, q), \nu=\alpha \beta^{-1}$, the operator $C_{b}^{*}$ is bounded from $L^{p}\left(\alpha^{p}\right)$ into $L^{q}\left(\beta^{q}\right)$, and the operator

$$
\text { p.v. } \int(b(x)-b(y)) k(x, y) f(y) d y
$$

exists a.e. for $f \in L^{p}\left(\alpha^{p}\right)$ and it is bounded from $L^{p}\left(\alpha^{p}\right)$ into $L^{q}\left(\beta^{q}\right)$,

(3.4) for any pair $\alpha, \beta \in A(p, q), \nu^{2}=\alpha \beta^{-1}$, the operator $C_{a, b}^{*}$ is bounded from $L^{p}\left(\alpha^{p}\right)$ into $L^{q}\left(\alpha^{q}\right)$, and the operator

$$
\text { p.v. } \int(a(x)-a(y))(b(x)-b(y)) k(x, y) f(y) d y
$$

exists a.e. for $f \in L^{p}\left(\alpha^{p}\right)$ and it is bounded from $L^{p}\left(\alpha^{p}\right)$ into $L^{q}\left(\beta^{q}\right)$.

The proof of (3.3) in the case $p=q$ can be found in [8]; here we shall give a sketch for the case (3.4).

Let $\phi, \psi \in \mathcal{C}^{\infty}([0, \infty))$ such that, $\left|\phi^{\prime}(t)\right| \leq C t^{-1},\left|\psi^{\prime}(t)\right| \leq C t^{-1}$ and

$$
\chi_{[2, \infty)} \leq \phi \leq \chi_{[1, \infty)}, \chi_{[1,2]} \leq \psi \leq \chi_{[1 / 2,3]} .
$$

We consider the operators

$$
\Phi f(x)=\left\{\phi_{\varepsilon} f(x)\right\}_{\epsilon>0}=\left\{\int k(x, y) \phi\left(\frac{|x-y|}{\varepsilon}\right) f(y) d y\right\}_{\varepsilon>0}
$$


and

$$
\Psi f(x)=\left\{\psi_{\varepsilon} f(x)\right\}_{\varepsilon>0}=\left\{\int|k(x, y)| \psi\left(\frac{|x-y|}{\varepsilon}\right) f(y) d y\right\}_{\epsilon>0},
$$

with kernels given by

$$
\left\{\phi_{\epsilon}(x, y)\right\}_{\varepsilon}=\left\{k(x, y) \phi\left(\frac{|x-y|}{\varepsilon}\right)\right\}_{\epsilon>0}
$$

and

$$
\left\{\psi_{\varepsilon}(x, y)\right\}_{\varepsilon}=\left\{|k(x, y)| \psi\left(\frac{|x-y|}{\epsilon}\right)\right\}_{\varepsilon>0} .
$$

The kernel of $\Phi$ as $\ell^{\infty}(\mathbf{R})$-valued function satisfies (K .2) of Theorem (2.3). Analogously, it can be shown that the kernel of $\Psi$ satisfies (W.3) of Theorem (2.6).

By the vector valued Calderon-Zygmund theory, see [5] and [6], $\Phi$ and $\Psi$ are bounded linear operators for $L^{p}$ into $L_{q \infty}^{q}, \frac{1}{p}-\frac{1}{q}=\frac{\gamma}{n}$. Therefore $\Phi$ satisfies the hypotheses of Theorem (2.3) and $\Psi$ the hypotheses of Theorem (2.6).

Let $\tilde{b}(x)=(b(x), b(x), \ldots, b(x), \ldots)$, it is clear that $\tilde{b} \in B M O_{\ell \infty}(\nu)$, and therefore by Theorem (2.3) and Theorem (2.6) the operators

$$
\begin{aligned}
& \Phi_{a, b} f(x)= \\
& \left\{a(x) b(x) \phi_{\varepsilon} f(x)-a(x) \phi_{\epsilon}(b f)(x)-b(x) \phi_{\epsilon}(a f)(x)+\phi_{\epsilon}(a b f)(x)\right\}_{c>0}
\end{aligned}
$$

and

$$
\Psi_{a, b}^{+} f(x)=\left\{\int|a(x)-a(y)||b(x)-b(y)| \psi_{\varepsilon}(x, y) f(y) d y\right\}_{\varepsilon>0}
$$

are bounded from $L^{p}\left(\alpha^{p}\right)$ into $L_{\ell^{\infty}}^{q}\left(\beta^{q}\right)$ for $\alpha, \beta \in A(p, q)$ and $\alpha \beta^{-1}=\nu^{2}$.

Now, we consider the operator

$$
\begin{aligned}
& \tilde{T}_{a, b} f(x)= \\
& \quad\left\{a(x) b(x) T_{\varepsilon} f(x)-a(x) T_{\varepsilon}(b f)(x)-b(x) T_{\varepsilon}(a f)(x)+T_{\epsilon}(a b f)(x)\right\}_{\varepsilon>0}
\end{aligned}
$$

The difference operator

$$
\begin{aligned}
& U_{a, b} f(x)=\Phi_{a, b} f(x)-\tilde{T}_{a, b} f(x)= \\
& \left\{\int(a(x)-a(y))(b(x)-b(y))\left[\phi\left(\frac{|x-y|}{\varepsilon}\right)-\chi[1, \infty)\left(\frac{|x-y|}{\varepsilon}\right)\right] k(x, y) f(y) d y\right\} \varepsilon>0
\end{aligned}
$$

satisfies, for a certain $\psi$ as above, that

$$
\begin{aligned}
& \left\|U_{a, b} f(x)\right\|_{\ell \infty} \leq \\
& \sup _{\varepsilon>0} \int\left|a(x)-a(y)\left\|b(x)-b(y)|| k(x, y)\left|\psi\left(\frac{|x-y|}{\varepsilon}\right)\right| f(y) \mid d y=\right\| \Psi_{a, b}^{+} f(x) \|_{\ell \infty}\right.
\end{aligned}
$$


and therefore $U_{a, b}$ is bounded from $L^{p}\left(\alpha^{p}\right)$ into $L_{\ell^{\infty}}^{q}\left(\beta^{q}\right)$ and, consequently, $\tilde{T}_{a, b}$ is bounded from $L^{p}\left(\alpha^{p}\right)$ into $L_{q \infty}^{q}\left(\beta^{q}\right)$, that is to say $\tilde{C}_{a, b}^{*}$ is bounded from $L^{p}\left(\alpha^{p}\right)$ into $L^{q}\left(\beta^{q}\right)$.

C. Let $p, 1<p<\infty ; \alpha$ and $\beta \in A_{p}, \nu^{2 p}=\alpha \beta^{-1}, a, b \in B M O(\nu)$, then the operator

$$
S_{a, b}^{*} f(x)=\sup _{r}\left|\int_{\mathbf{R}} \frac{(b(x)-b(y))(a(x)-a(y))}{x-y} e^{-i r y} f(y) d y\right|,
$$

is bounded from $L^{p}(\alpha)$ into $L^{p}(\beta)$.

To prove this it is enough to observe that, by the Carleson-Hunt theorem, see [4], the operator

$$
S f(x)=\left\{\int_{\mathbf{R}} \frac{e^{-i r y}}{x-y} f(y) d y\right\} .
$$

is bounded from $L^{p}(\mathbf{R})$ into $L_{k \infty}^{p}(\mathbf{R})$, for any $p, 1<p<\infty$. The kernel of this operator satisfles (K.1) and (K.2), see [5], therefore it is enough to apply theorem 1 with $\tilde{b}(x)=(b(x), b(x), \ldots, b(x), \ldots)$.

D. Let $H$ be the Hilbert transform

$$
H f(x)=p . v . \int \frac{f(y)}{x-y} d y,
$$

and let $E$ be a U.M.D. Banach space, see [2]. Let $p, 1<p<\infty, \alpha$ and $\beta \in A_{p}, \nu^{2}=\left(\alpha \beta^{-1}\right)^{1 / p}$ and $a, b \in B M O_{\alpha(E . E)}(\nu)$. Moreover, we assume thint $\alpha(x) b(y)=b(y) a(x)$ holds for every $x, y \in \mathbf{R}^{n}$. Then the operator

$$
p . v . \int \frac{(a(x)-a(y))(b(x)-b(y))}{x-y} f(y) d y,
$$

is bounder f from $L_{t}^{p}(\alpha)$ into $L_{k}^{p}(\beta)$.

E. Let, $I_{\gamma}$, $\alpha$ the fructional integral, of order $\gamma$,

$$
I_{-} f(x)=\int \frac{f(y)}{|x-y|^{n-\gamma}} d y, \quad, 0<\gamma<n .
$$

It is known, sox $\{6\}$, that $I_{x}$ is lrancled from $L_{f}^{p}\left(\mathbf{R}^{n}\right)$ into $L_{E}^{q}\left(\mathbf{R}^{n}\right)$, for any Bankach spact $E$ and $\frac{1}{p}-\frac{1}{q}=\frac{7}{n}$. Let $\alpha, \beta \in A(p, q), \nu^{2}=\alpha \beta^{-1}$ and $a, b \in B M O_{C(N, b)}(\nu)$. Morerver, wo iassume that, $a(x) b(y)=b(y) a(x)$ holds for every $x, y \in \mathbf{R}^{n}$. Then the operators

$$
\left[\left[I_{\gamma}, b\right] a\right] f(x)=\int \frac{(a(x)-a(y))(b(x)-b(y))}{|x-y|^{n-\gamma}} f(y) d y
$$


and

$$
I_{\gamma, a, b}^{+} f(x)=\int \frac{|a(x)-a(y)||b(x)-b(y)|}{|x-y|^{n-\gamma}} f(y) d y,
$$

are bounded from $L_{E}^{p}\left(\alpha^{p}\right)$ into $L_{E}^{q}\left(\beta^{q}\right)$ for any Banach space $E$.

F. Maximal operators. Let $0 \leq \gamma<n$. Suppose that $\phi \in L^{\frac{n}{n-\gamma}}\left(\mathbf{R}^{n}\right)$ and verifies

$$
|\phi(x-y)-\phi(x)| \leq C|y||x|^{-n-1+\gamma} \text {, when }|x|>2|y| .
$$

Set $\phi_{\varepsilon}(x)=\varepsilon^{-n+\gamma} \phi\left(\varepsilon^{-1} x\right)$. Then the operator

$$
\left.M_{\phi} f(x)\right)=\left\{f * \phi_{\varepsilon}(x)\right\}_{e}>0,
$$

can be viewed as a vector-valued Calderón-Zygmund operator, bounded from $L^{p}\left(\mathbf{R}^{n}\right)$ into $L_{q \infty}^{q}\left(\mathbf{R}^{n}\right), \frac{1}{p}-\frac{1}{q}=\frac{\gamma}{n}$, see $[5]$ and [6]. Therefore proceeding as in application $\mathrm{C}$ we have that the operators

$$
M_{\phi, b} f(x)=\left\{b(x) f * \phi_{\varepsilon}(x)-(b f) * \phi_{e}(x)\right\}_{\epsilon>0}
$$

and

$$
M_{\phi, b}^{+} f(x)=\left\{\int|b(x)-b(y)| \phi_{\epsilon}(x-y) f(y) d y\right\}_{\epsilon>0},
$$

are bounded from $L^{p}\left(\alpha^{p}\right)$ into $L_{\ell^{\infty}}^{q}\left(\beta^{q}\right), \frac{1}{p}-\frac{1}{q}=\frac{\gamma}{n}$, where $\alpha$ and $\beta \in A(p, q)$, $\alpha \beta^{-1}=\nu$ and $b \in B . M . O(\nu)$; also we have that the operators

$$
\begin{aligned}
M_{\phi, a, b} f(x)= & \left\{a(x) b(x)\left[f * \phi_{\varepsilon}\right](x)-a(x)\left[(b f) * \phi_{\varepsilon}\right](x)\right. \\
& \left.-b(x)\left[(a f) * \phi_{\varepsilon}\right](x)+\left[(a b f) * \phi_{\varepsilon}\right](x)\right\}
\end{aligned}
$$

and

$$
M_{\phi, a, b}^{+} f(x)=\left\{\int|b(x)-b(y) \|| a(x)-a(y) \mid \phi_{\epsilon}(x-y) f(y) d y\right\}_{\epsilon>0}
$$

are bounded from $L^{p}\left(\alpha^{p}\right)$ into $L_{l^{\infty}}^{q}\left(\beta^{q}\right), \frac{1}{p}-\frac{1}{q}=\frac{\gamma}{n}$, where $\alpha$ and $\beta \in A(p, q)$, $\alpha \beta^{-1}=\nu^{2}, b$ and $a$ belong to $B M O(\nu)$.

It is clear that choosing $\phi$ as above and such that $\chi_{[-1,1]} \leq \phi$ we car deduce that the operators

$$
\begin{aligned}
S_{b}^{+} f(x) & =\sup _{x \in Q} \frac{1}{|Q|^{1-\frac{x}{n}}} \int_{Q}|b(x)-b(y)| f(y) d y, \\
S_{b} f(x) & =\sup _{x \in Q} \frac{1}{|Q|^{1-\frac{\gamma}{n}}} \int_{Q}(b(x)-b(y)) f(y) d y, \\
S_{a, b}^{+} f(x) & =\sup _{x \in Q} \frac{1}{|Q|^{1-\frac{x}{n}}} \int_{Q}|a(x)-a(y) \| b(x)-b(y)| f(y) d y, \text { and } \\
S_{a, b} f(x) & =\sup _{x \in Q} \frac{1}{|Q|^{1-\frac{\gamma}{n}}} \int_{Q}(a(x)-a(y))(b(x)-b(y)) f(y) d y,
\end{aligned}
$$

satisfy the analogous boundedness properties. In fact we have the following theorem 
(3.5) Theorem. Let $\nu$ be a weight in $A_{2}$ such that $\nu^{\frac{n}{n-\gamma}} \in A_{2}$. Then the following conditions are equivalent

(a) For $\frac{1}{p}-\frac{1}{q}=\frac{\gamma}{n}, \alpha$ and $\beta \in A(p, q)$ and $\nu=\alpha \beta^{-1}$, the operator $S_{b}^{+}$maps $L^{p}\left(\alpha^{p}\right)$ into $L^{q}\left(\beta^{q}\right)$.

(b) For $\frac{1}{p}-\frac{1}{q}=\frac{\gamma}{n}, \alpha$ and $\beta \in A(p, q)$ and $\nu=\alpha \beta^{-1}$, the operator $S_{o}$ maps $L^{p}\left(\alpha^{p}\right)$ into $L^{q}\left(\beta^{q}\right)$.

(c) If $\frac{n}{n-\gamma}=\frac{q_{0}}{2}, \nu^{q_{0} / 2}=\nu_{0} \nu_{1}^{-1}$ with $\nu_{0}$ and $\nu_{1} \in A_{1}$, then $S_{b}$ maps $L^{p_{0}}\left(\left(\nu_{0} \nu_{1}^{-1}\right)^{p_{0} / 40}\right)$ into $L^{q_{0}}\left(\nu_{0}^{-1} \nu_{1}\right)$ for $\frac{1}{p_{0}}-\frac{1}{q_{0}}=\frac{\gamma}{n}$.

(d) b belongs to B.M.O.(L).

Proof: We have scen that $(d) \Rightarrow(a)$ and it is obvious that $(a) \Rightarrow(b)$.

To see that $(b) \Rightarrow$ (c) observe that with this election of $q_{0}$ we have $p_{0}^{\prime}=q_{0}$ and $\beta=\left(\nu_{0}^{-1} \nu_{1}\right)^{1 / q_{0}} \in A(p, q), \alpha=\left(\nu_{0} \nu_{1}^{-1}\right)^{1 / q_{0}} \in A(p, q)$, and $\alpha \beta^{-1}=\nu$.

Now we prove $(c) \Rightarrow(d)$.

$$
\begin{aligned}
\int_{Q}\left|b(x)-b_{Q}\right| d x=|Q|^{-\gamma / n} \int_{Q}\left|\frac{1}{|Q|^{1-\gamma / n}} \int_{Q}(b(x)-b(y)) d y\right| d x \\
\leq|Q|^{-\gamma / n}\left(\int_{Q}\left|\frac{1}{|Q|^{1-\gamma / n}} \int_{Q}(b(x)-b(y)) d y\right|^{q_{0}} \nu_{0}^{-1}(x) \nu_{1}(x) d x\right)^{1 / q_{0}} \\
\\
\cdot\left(\int_{Q}\left(\nu_{0}(x) \nu_{1}^{-1}(x)\right)^{q_{0}^{\prime} / q_{0}}\right)^{1 / q_{0}^{\prime}} \\
\leq C|Q|^{-\gamma / n}\left(\int_{Q}\left(\nu_{0}(x) \nu_{1}^{-1}(x)\right)^{p_{0} / q_{0}} d x\right)^{1 / p_{0}}\left(\int\left(\nu_{0}(x) \nu_{1}^{-1}(x)\right)^{q_{0}^{\prime} / q_{0}} d x\right)^{1 / q_{0}^{\prime}} \\
=C|Q|^{-\gamma / n}\left(\int_{Q}\left(\nu(x)^{q_{0} / 2}\right)^{p_{0} / q_{0}} d x\right)^{2 / p_{0}} \leq C|Q|^{-\gamma / n}\left(\int_{Q} \nu(x)^{p_{0} / 2}\right)^{2 / p_{0}} \\
\leq C|Q|^{-\gamma / n}\left(\int_{Q} \nu(x) d x\right)|Q|^{\left(2 / p_{0}\right) /\left(2 / p_{0}\right)^{\prime}}=C\left(\int_{Q} \nu(x) d x\right) \cdot \mathbf{\square}
\end{aligned}
$$

\section{Proofs of the commutator theorems}

(4.1) Definition. Let $1 \leq s<\infty, E$ be a Banach space, $\nu \in A_{2}, \alpha, \beta$ positive functions, $a$ and $b$ functions belonging to $B M O_{C(E, E)}(\nu)$ and $f$ be an $\mathrm{E}$-valued function. We define the following maximal functions.

$$
\begin{gathered}
M_{1} f(x)=\sup \frac{1}{|Q|} \int_{Q}\left\|\left(b(y)-b_{Q}\right) f(y)\right\| d y \\
M_{2}^{s} f(x)=\sup |Q|^{\gamma / n}\left(\frac{1}{|Q|} \int_{Q}\left\|\left(b(y)-b_{Q}\right) f(y)\right\|^{s} d y\right)^{1 / s}
\end{gathered}
$$




$$
M_{3} f(x)=\sup \left(\inf _{y \in Q}\left(\nu \mathcal{X}_{Q}\right)^{*}(y)\right)\left(|Q|^{\gamma / n} \frac{1}{|Q|} \int_{Q}\|f(y)\| d y\right)
$$

$$
M_{4} f(x)=\sup \frac{1}{|Q|} \int_{Q}\left\|\left(a(y)-a_{Q}\right) f(y)\right\| d y
$$

$$
\begin{gathered}
M_{5}^{s} f(x)=\sup |Q|^{\gamma / n}\left[\frac{1}{|Q|} \int_{Q}\left\|\left(a(y)-a_{Q}\right) f(y)\right\|^{s} \alpha^{s / 2}(y) d y\right]^{1 / s} \\
{\left[\frac{1}{|Q|} \int_{Q}\left\|b(y)-b_{Q}\right\|^{s} \alpha^{-s / 2}(y) d y\right]^{1 / s}}
\end{gathered}
$$

(4.7) $M_{6}^{s} f(x)=\sup |Q|^{\gamma / n}\left[\frac{1}{|Q|} \int_{Q}\left\|\left(a(y)-a_{Q}\right)\left(b(y)-b_{Q}\right) f(y)\right\|^{s} d y\right]^{1 / s}$,

(4.8) $M_{7} f(x)=\sup \left(\inf _{y \in Q}\left(\nu \mathcal{X}_{Q}\right)^{*}(y)\right)\left(\frac{|Q|^{\gamma / n}}{|Q|} \int_{Q}\left\|\left(b(y)-b_{Q}\right) f(y)\right\| d y\right)$

(4.9) $M_{8} f(x)=\sup \left(\inf _{y \in Q}\left(\nu \mathcal{X}_{Q}\right)^{*}(y)\right)\left(\frac{|Q|^{\gamma / n}}{|Q|} \int_{Q}\left\|\left(a(y)-a_{Q}\right) f(y)\right\| d y\right)$,

$$
M_{9} f(x)=\sup \left(\inf _{y \in Q}\left(\nu X_{Q}\right)^{*}(y)\right)^{2}\left(\frac{|Q|^{\gamma / n}}{|Q|} \int_{Q}\|f(y)\| d y\right)
$$

$$
\begin{aligned}
M_{10}^{j} f(x) & =\sup \left(\frac{1}{|Q|} \int_{Q}\left\|a(y)-a_{Q}\right\| d y\right)\left(\frac{1}{|Q|} \int_{Q}\left\|b(y)-b_{Q}\right\| d y\right) \\
& \left(\frac{\left|2^{j} Q\right|^{\gamma / n}}{\left|2^{j} Q\right|} \int_{2^{i} Q}\|f(y)\| d y\right),
\end{aligned}
$$

$$
\begin{aligned}
M_{11}^{j} f(x) & =\sup \left(\inf _{y \in 2^{i} Q}\left(\nu \mathcal{X}_{2}{ }\right)^{*}(y)\right)\left(\frac{1}{|Q|} \int_{Q}\left\|b(y)-b_{Q}\right\| d y\right) \\
& \left(\frac{\left|2^{j} Q\right|^{\gamma / n}}{\left|2^{3} Q\right|} \int_{2^{\prime}{ }_{Q}}\|f(y)\| d y\right),
\end{aligned}
$$


(4.13) $M_{12}^{j} f(x)=$

$$
\sup \left(\frac{1}{|Q|} \int_{Q}\left\|b(y)-b_{Q}\right\| d y\right)\left(\frac{\left|2^{j} Q\right|^{\gamma / n}}{\left|2^{j} Q\right|} \int_{2^{i} Q}\left\|\left(a_{2^{i} Q}-\alpha(y)\right) f(y)\right\| d y\right),
$$

$$
M_{13} f(x)=\sup \left(\frac{1}{|Q|} \int_{Q}\left\|b(y)-b_{Q}\right\| d y\right)\left(\frac{1}{|Q|} \int_{Q}\|f(y)\| d y\right)
$$

$$
\begin{aligned}
M_{14}^{s} f(x)= & \sup \left(\frac{1}{|Q|} \int_{Q}\left\|b(y)-b_{Q}\right\| d y\right)|Q|^{\gamma / n}\left(\frac{1}{|Q|} \int_{Q}\|f(y)\|^{s} \alpha^{s / 2}(y) d y\right)^{1 / s} \\
& \left(\frac{1}{|Q|} \int_{Q} \beta^{-s / 2}(y) d y\right)^{1 / s} .
\end{aligned}
$$

In all the cases the supremum is taken over all cubes in $\mathbf{R}^{n}$ with sides paralell to the axes and centered in $x .\left(\nu \mathcal{X}_{\Omega}\right)^{*}$ stands for the Hardy-Littlewood maximal function of $\nu \mathcal{X}_{Q}$.

(4.16) Proposition. Let $E$ be a Banach space. Let $0 \leq \gamma<n$, assume $\alpha^{-n /(n-\gamma)}$ and $\beta^{-n /(n-\gamma)} \in A_{1}, \nu=\alpha \beta^{-1}$ and $b \in B M O_{\mathcal{C}\left(E_{1} E\right)}(\nu)$. Then

$$
\left\|\beta M_{1} f\right\|_{L^{\infty}} \leq C\|f \alpha\|_{L_{E}^{\infty}},
$$

(4.18) There exists $\varepsilon>0$ such that if $1 \leq s<(1+\varepsilon)$ then

$$
\left\|\beta M_{2}^{g} f\right\|_{L^{\infty}} \leq C\|f \alpha\|_{L_{E}^{n / \gamma}} \quad \text { and }
$$

$$
\left\|\beta M_{3} f\right\|_{1, \infty} \leq C\|f \alpha\|_{L_{E}^{n / \gamma}}
$$

(4.20) Proposition. Let $E$ be a Banach space. Let $0 \leq \gamma<n$, assume $\alpha^{-n /(n-\gamma)}, \delta^{-n / n-\gamma}$, and $\beta^{-n /(n-\gamma)} \in A_{1}, \nu^{2}=\alpha \beta^{-1}, \nu=\alpha \delta^{-1}=\delta \beta^{-1}$, and $a, b \in B M O_{\mathcal{L}(E, E)}$. Then

$$
\left\|\beta M_{i} f\right\|_{L^{\infty}} \leq C\|f \alpha\|_{L_{E}^{n / \gamma}}, \quad i=7,8,9, \quad \text { and }
$$

$$
\left\|\beta M_{i}^{j} f\right\|_{L^{\infty}} \leq C\|f \alpha\|_{L_{E}^{n / \gamma}}, \quad i=10,11,12 . \quad j \geq 1
$$


(4.23) If $u=\frac{2 n}{n+\gamma}$

$$
\left\|\beta M_{s}^{u} f\right\|_{L^{\infty}} \leq C\|f \alpha\|_{L_{E}^{n / \gamma}} .
$$

(4.24) There exists $\varepsilon>0$ such that if $1 \leq s<(1+\varepsilon)$ then

$$
\left\|\beta M_{6}^{s} f\right\|_{L^{\infty}} \leq C\|f \alpha\|_{L_{E}^{n / \gamma}} \quad \text { and }
$$

$$
\left\|\beta M_{i} f\right\|_{L^{\infty}} \leq C\|f \delta\|_{L_{E}^{\infty}, i=4,13}
$$

(4.26) If $u=\frac{2 n}{n+\gamma}$

$$
\left\|\beta M_{14}^{u} f\right\|_{L^{\infty}} \leq C\|f \alpha\|_{L_{E}^{n / \gamma}} .
$$

We postpone the proofs of these Propositions. Now we state and prove the following Corollaries.

(4.27) Corollary. Let $\nu^{-\frac{n}{n-7}} \in A_{2}, \quad 0 \leq \gamma<n$. Then in the hypothesis of Proposition (4.16) we have that

(4.28) if $\alpha, \beta \in A_{p}, 1<p<\infty$, and $\alpha \beta^{-1}=\nu^{p}$ then

$$
\left\|M_{1} f\right\|_{L^{\nabla}(\beta)} \leq C\|f\|_{L_{E}^{p}\{\alpha\rangle},
$$

(4.29) if $\alpha, \beta \in A(p, q), \frac{1}{p}-\frac{1}{q}=\frac{\gamma}{n}$ and $\alpha \beta^{-1}=\nu$ then

$$
\left\|M_{2}^{s} f\right\|_{L^{q}\left(\beta^{\natural}\right)} \leq C\|f\|_{L_{E}^{p}\left(\alpha^{\mathcal{D}}\right)}, 1 \leq s<(1+\varepsilon)
$$

and

$$
\left\|M_{3} f\right\|_{L q\left(\beta^{\imath}\right)} \leq C\|f\|_{L_{E}^{p}\left(\alpha^{p}\right)} .
$$

(4.30) Corollary. Let $\nu^{-\frac{2 n}{n-\gamma}} \in A_{2}, 0 \leq \gamma<n$. Then in the hypothesis of Proposition (4.20) we have that

(4.31) if $\alpha, \beta \in A(p, q), \frac{1}{p}-\frac{1}{q}=\frac{\gamma}{n}$, and $\alpha \beta^{-1}=\nu^{2}$, then

$$
\begin{gathered}
\left\|M_{\mathrm{i}} f\right\|_{L^{\natural}\left(\beta^{\natural}\right)} \leq C\|f\|_{L_{\varepsilon}^{p}\left(\alpha^{p}\right)} i=7,8,9, \\
\left\|M_{i}^{j} f\right\|_{L^{q}\left(\beta^{\natural}\right)} \leq C\|f\|_{L_{E}^{p}\left(\alpha^{p}\right)} i=10,11,12,, j \geq 1, \\
\left\|M_{i}^{u} f\right\|_{L^{q}\left(\beta^{q}\right)} \leq C\|f\|_{L_{E}^{p}\left(\alpha^{p}\right)}, u=\frac{2 n}{n+\gamma}, i=5,14
\end{gathered}
$$

and

$$
\left\|M_{6}^{s} f\right\|_{L^{q}\{\beta q\}} \leq C\|f\|_{L_{\varepsilon}^{p}\left(\alpha^{p}\right)}, 1 \leq s<(1+\varepsilon)
$$


(4.32) if $\delta, \beta \in A_{p}, 1<p<\infty$ and $\delta \beta^{-1}=\nu^{p}$, then

$$
\left\|M_{i} f\right\|_{L p(\beta)} \leq C\|f\|_{L_{E}^{p}(\delta)}, i=4,13
$$

For the proof of these Corollarics it is enough to observe that for a sublinear operator $S$, the inequality

$$
\|S f\|_{L^{D}(\beta)} \leq C\|f\|_{L^{p}(\alpha)} \alpha, \beta \in A_{p} \quad \text { and } \quad \alpha \beta^{-1}=\nu^{p}
$$

is equivalent to the inequality

$$
\|U(g)\|_{L^{p}(w)} \leq C\left\|_{g}\right\|_{L P(w)}, w \in A_{p}^{(\nu)},
$$

$U$ being the operator $U(g)=S\left(g \nu^{-1}\right)$.

Analogously, observe that the inequality

$$
\|S f\|_{L^{Q}(\beta q)} \leq C\|f\|_{L^{p}\left(\alpha^{p}\right)}, \alpha, \beta \in A(p, q) \text { and } \alpha \beta^{-3}=\nu,
$$

is equivalent to the inequality

$$
\|U(g)\|_{L^{Q}(w q)} \leq C\|g\|_{L^{p}\left(w^{p}\right)}, w \in A^{(\nu)}(p, q),
$$

$U$ being the operator $U(g)=S\left(g \nu^{-1}\right)$.

With these two observations the corollaries (4.27) and (4.30) are direct consequences of Theorems (1.2) and (1.3).

(4.33) Proposition. There exists $\varepsilon>0$, such that if

$$
I \leq s<(1+\varepsilon), \text { and } u=\frac{2 n}{n+\gamma},
$$

then the operators considered in Theorem (2.3) and in Theorem (2.6) satisfy the following inequalities

$$
\left(C_{b} f\right)^{\#}(x) \leq C\left\{M_{1}(T f)(x)+M_{2}^{y} f(x)+M_{3} f(x)\right\}
$$

$$
\begin{aligned}
\left(C_{a, b} f\right)^{\#}(x) & \leq C\left\{M_{4}\left(C_{b} f\right)(x)+M_{13}\left(C_{a} f\right)(x)\right. \\
& +M_{5}^{u} f(x)+M_{6}^{s} f(x)+M_{7} f(x)+M_{8} f(x)+M_{9} f(x) \\
& +\sum_{j=1}^{\infty} 2^{-j}\left(M_{10}^{j} f(x)+M_{12}^{j} f(x)\right)+\sum_{j=1}^{\infty} j 2^{-j} M_{11}^{j} f(x) \\
& \left.+M_{14}^{u} f(x)\right\} \quad \text { and }
\end{aligned}
$$


(4.37)

$$
\begin{aligned}
\left(V_{a, b}^{+} f\right)^{\#}(x) & \leq C\left\{M_{4}\left(V_{b}^{+} f\right)(x)+M_{13}\left(V_{a}^{+} f\right)(x)\right. \\
& +M_{5}^{u} f(x)+M_{6}^{s} f(x)+M_{7} f(x)+M_{8} f(x)+M_{9} f(x) \\
& +\sum_{j=1}^{\infty} 2^{-j}\left(M_{10}^{j} f(x)+M_{12}^{j} f(x)\right)+\sum_{j=1}^{\infty} j 2^{-j} M_{11}^{j} f(x) \\
& \left.+M_{14}^{u} f(x)\right\} .
\end{aligned}
$$

Assuming this Proposition (4.33) we can give the proof of Theorem (2.3) and Theorem (2.6). We prove Theorem (2.3) only, since the proof of Theorem (2.6) is similar.

In fact, we shall give only the proof of (2.5) assuming that (2.4) is true. The proof of (2.4) is similar using remark (2.10).

By (4.36) and Corollary (4.30) we have

$$
\begin{aligned}
& \left(\int\left(C_{a, b} f\right)^{\#}(x)^{q} \beta^{q}(x) d x\right)^{1 / q} \leq C\left\{\left(\int\left\|C_{b} f(x)\right\|^{q} \delta^{q}(x) d x\right)^{1 / q}\right. \\
& \left.+\left(\int\left\|C_{a} f(x)\right\|^{q} \delta^{q}(x) d x\right)^{1 / q}+\left(\int\|f(x)\|^{p} \alpha^{p}(x) d x\right)^{1 / p}\right\}
\end{aligned}
$$

Then by (2.4) and the vector-valued version of the sharp function theorem, see $[5]$, we have

$$
\begin{aligned}
\left(\int\left\|C_{a, b} f(x)\right\|^{q} \beta^{q}(x) d x\right)^{1 / q} & \leq C\left(\int\left(C_{a, b} f\right)^{\#}(x)^{q} \beta^{q}(x) d x\right)^{1 / q} \\
& \leq C\left(\int\|f(x)\|^{p} \alpha^{p}(x) d x\right)^{1 / p} .
\end{aligned}
$$

This ends the proof of section (2.5) in Theorem (2.3).

Now we give the proofs of the technical propositions (4.16), (4.20) and (4.33). We shall need the following lemmas.

(4.38) Lemma. Let $E$ be a Banach space. Let $Q$ be a cube and $Q_{k}=2^{k} Q$. Then if $b \in B M O_{E}(\nu), \nu \in A_{2}$, it follows that

$$
\left\|b_{Q}-b_{Q_{k}}\right\| \leq C k \nu_{Q_{i(k)}} \leq k C \inf _{y \in Q_{k}}\left(\nu \mathcal{X}_{Q_{k}}\right)^{*}(y)
$$

where $Q_{i(k)}$ is the cube such that $\nu_{Q_{i(k)}}=\max _{1 \leq j \leq k} \nu_{Q_{j}}$ and $\left(\nu \mathcal{X}_{Q_{k}}\right)^{*}$ is the HardyLittlewood maximal function of $\nu \mathcal{X}_{Q_{k}}$.

(4.39) Lemma. If $w^{-t} \in A_{1}$, there exists $\varepsilon>0$ such that for every $1 \leq r \leq$ $t(1+\varepsilon), w^{-r} \in A_{1}$. 
(4.40) Lemma. If $w^{-t} \in A_{1}$, there exists $\varepsilon>0$ such that $w^{r^{\prime}} \in A_{\mathrm{r}^{\prime} / t}$ for every $1<\tau \leq(1+\varepsilon)$.

(4.41) Lemma. Let $E$ be a Banach space, if $b \in B M O_{E}(\nu)$ and $\nu^{l}=$ $\alpha \beta^{-1}, \alpha^{-t} \in A_{1}, \beta^{-t} \in A_{1}$ then there exists $\varepsilon>0$ such that

$$
\left(\frac{1}{|Q|} \int_{Q}\left\|b(x)-b_{Q}\right\|^{i r} \alpha^{-r}(x) d x\right)^{1 / r} \leq C \beta\left(x_{0}\right)^{-1}
$$

holds for $1<r<t(1+\varepsilon)$ and $x_{0} \in Q, l=1,2$.

The proof of these lemmas can be found in [1].

(4.42) Lemma. Let $E$ be a Banach space; $0 \leq \gamma<n, \alpha^{-\frac{n}{n-\gamma}}, \beta^{-\frac{n}{n-\gamma}} \in A_{1}$, $\nu=\alpha \beta^{-1}$ and $b \in B M O_{\mathcal{L}(E, E)}(\nu)$. Then for any function $f$ we have,

$$
\text { if } 1 \leq p<\frac{n}{\gamma} \text { then, }\left(\frac{1}{|Q|} \int_{Q}\|f \alpha\|^{p} d x\right)^{1 / p} \leq\|f \alpha\|_{n / \gamma}|Q|^{-\gamma / n} \text {, }
$$

(4.44) there exists $\varepsilon>0$ such that if $1 \leq s<(1+\varepsilon)$, then,

$$
\left(\frac{1}{|Q|} \int_{Q}\left\|\left(b-b_{Q}\right) f\right\|^{s} d x\right)^{1 / s} \leq C\|f \alpha\|_{n / \gamma}|Q|^{-\gamma / n}\left(i n f_{x \in Q} \beta^{-1}(x)\right)
$$

(4.45) there exists $\varepsilon>0$ such that if $1 \leq s<\frac{n}{n-\gamma}(1+\varepsilon)$, then,

$$
\begin{aligned}
\frac{1}{|Q|} \int_{Q}\left\|\left(b-b_{Q}\right) f\right\| d x & \leq\left(\frac{1}{|Q|} \int_{Q}\left\|b-b_{Q}\right\|^{s} \alpha^{-s} d x\right)^{1 / s}\|f \alpha\|_{\infty} \\
& \leq C\left(\inf _{x \in Q} \beta^{-1}(x)\right)\|f \alpha\|_{\infty} \quad \text { and }
\end{aligned}
$$

$$
\left\|\frac{1}{|Q|} \int_{Q} f d x\right\| \leq C\|f \alpha\|_{n / \gamma}|Q|^{-\gamma / n}\left(i n f_{x \in Q} \alpha^{-1}(x)\right)
$$

Proof: (4.43) is obvious by using Hölder's inequality. Lemma (4.41) and Holder's inequality give (4.45). In order to prove (4.46) observe that

$$
\left\|\frac{1}{|Q|} \int_{Q} f d x\right\| \leq\left(\frac{1}{|Q|} \int_{Q}\|f \alpha\|^{p} d x\right)^{1 / p}\left(\frac{1}{|Q|} \int_{Q} \alpha^{-p^{\prime}}\right)^{1 / p^{\prime}}
$$


Choosing $p, 1 \leq p<\frac{n}{\gamma}$, such that $p^{\prime}<\left(\frac{n}{n-\gamma}\right)(1+\varepsilon)$ and $\alpha^{-p^{\prime}} \in A_{1}$, then (4.43) gives the result. Finally by Holder's inequality we have in (4.44) that

$$
\begin{aligned}
& \left(\frac{1}{|Q|} \int_{Q}\left\|\left(b-b_{Q}\right) f\right\|^{s} d x\right)^{1 / s} \\
& \leq\left(\frac{1}{|Q|} \int_{Q}\left\|\left(b-b_{Q}\right)\right\|^{s t} \alpha^{-s t} d x\right)^{1 / s t}\left(\frac{1}{|Q|} \int_{Q}\|f \alpha\|^{s t^{\prime}} d x\right)^{1 / s t^{\prime}} .
\end{aligned}
$$

Now if we choose $t$ such that $s t<\frac{n}{n-\gamma}(1+\varepsilon)$ and $s t^{\prime}<\frac{n}{\gamma}$, where $\varepsilon$ is the one which appears in lemma (4.41), we get that the last product is less than

$$
C\|f \alpha\|_{\frac{n}{\gamma}}|Q|^{-\gamma / n} \text { in } f_{x \in Q} \beta^{-1}(x) .
$$

(4.47) Lemma. Let $t \geq 1$, and $\omega^{-t} \in A_{1}$, then $\omega^{1 / 2} \in A\left((2 t)^{\prime}, 2 t\right)$.

Proof of Proposition (4.16): Through this proof "sup "always shall mean the supremum over the cubes centered at $x$. The proof of (4.17) and (4.18) are direct applications of (4.45) and (4.44).

To show (4.19), choose $r$ such that $\frac{n}{n-\gamma}<r<\frac{n}{(n-\gamma)}(1+\varepsilon), r^{\prime}<\frac{n}{\gamma}$ and $\alpha^{-r} \in A_{1}$ then by (4.43), $M_{3} f(x)$ is less than

$$
\begin{aligned}
& \sup \left(\inf _{y \in Q}\left(\nu \mathcal{X}_{Q}\right)^{*}(y)\right)|Q|^{\gamma / n}\left(\frac{1}{|Q|} \int_{Q}\|f(y) \alpha(y)\|^{r^{\prime}} d y\right)^{1 / r^{\prime}}\left(\frac{1}{|Q|} \int_{Q} \alpha^{-r}(y) d y\right)^{1 / r} \\
& \leq C \sup \left(\inf _{y \in Q}\left(\nu \mathcal{X}_{Q}\right)^{*}(y)\right)\|f \alpha\|_{\frac{n}{\gamma}}\left(i n f_{y \in Q} \alpha^{-1}(y)\right) \\
& \leq C \sup \|f \alpha\|_{\frac{n}{\gamma}} \inf _{y \in Q}\left(\left(\inf _{z \in Q} \alpha^{-1}(z)\right) \cdot\left(\nu \mathcal{X}_{Q}\right)^{*}(y)\right) \leq C\|f \alpha\|_{\frac{n}{\gamma}} \beta^{-1}(x) .
\end{aligned}
$$

Proof of Proposition (4.20): Through this proof the word "sup "always shall mean the supremun over the cubes centered at $x$. Let $\alpha \delta^{-1}=\nu=\delta \beta^{-1}$. have

If $u=\frac{2 n}{n+\gamma}$, we have that $u\left(\frac{n / \gamma}{u}\right)^{\prime}=\frac{2 n}{n-\gamma}$, then by Hölder's inequality, we

$$
\begin{aligned}
M_{5}^{u} f(x) & \leq \sup \|f \alpha\|_{n / \gamma}\left(\frac{1}{|Q|} \int_{Q}\left\|a(y)-\alpha_{Q}\right\|^{2 n / n-\gamma} \alpha^{-n / n-\gamma}(y) d y\right)^{(n-\gamma) / 2 n} \\
& \left(\frac{1}{|Q|} \int_{Q}\left\|b(y)-b_{Q}\right\|^{u} \alpha^{-u / 2}(y) d y\right)^{1 / u}
\end{aligned}
$$


Observe that $\frac{u}{2}<\frac{n}{n-\gamma}$, then applying lemma (4.41) we get

$$
M_{5}^{u} f(x) \leq C\|f \alpha\|_{n / \gamma} \beta^{-1 / 2}(x) \beta^{-1 / 2}(x)=C\|f \alpha\|_{n / \gamma} \beta^{-1}(x) .
$$

If $1 \leq s<(1+\varepsilon)$, then by Hölder's inequality, we have

$$
\begin{aligned}
M_{6}^{s} f(x) & \leq \sup |Q|^{\gamma / n}\left(\frac{1}{|Q|} \int_{Q}\left\|\left(\alpha(y)-a_{Q}\right)\left(b(y)-b_{q}\right)\right\|^{s t^{\prime}} \alpha^{-s t^{t}}(y) d y\right)^{1 / s t^{\prime}} \\
& \left(\frac{1}{|Q|} \int_{Q}\|f(y)\|^{s t} \alpha^{s t}(y) d y\right)^{1 / s t} .
\end{aligned}
$$

Now if we choose $t$ such that $s t^{\prime}<\frac{n}{n-\gamma}(1+\varepsilon)$ and $s t<\frac{n}{\gamma}$, where $\varepsilon$ is the one which appears in lemma (4.41) we get

$$
M_{6}^{s} f(x) \leq \sup \int_{Q}\left(\left\|\left(a(y)-a_{Q}\right)\left(b(y)-b_{Q}\right)\right\|^{s t^{\prime}} \alpha^{-s t^{\prime}}(y) d y\right)^{1 / s t^{\prime}}\|f \alpha\|_{n / \gamma}
$$

Now by Hölder's inequality and lemma (4.41), we have,

$$
\begin{aligned}
& M_{6}^{s} f(x) \leq \sup \left(\frac{1}{|Q|} \int_{Q}\left\|a(y)-a_{Q}\right\|^{2 s t^{t}} \alpha^{-s t^{\prime}}(y) d y\right)^{1 / 2 s t^{\prime}} \\
& \left(\frac{1}{|Q|} \int_{Q}\left\|b(y)-b_{Q}\right\|^{2 s t^{\prime}} \alpha^{-s t^{\prime}}(y) d y\right)^{1 / 2 s t^{\prime}}\|f \alpha\|_{n / \gamma} \leq C \beta^{-1}(x)\|f \alpha\|_{n / \gamma} .
\end{aligned}
$$

Using (4.44) we get,

$$
\begin{aligned}
M_{7} f(x) & \leq C \sup \left(\inf _{y \in Q}\left(\nu \mathcal{X}_{Q}\right)^{*}(y)\right)\|f \alpha\|_{\frac{n}{7}} \text { in } f_{y \in Q} \delta^{-1}(y) \\
& \leq C \sup \left(\inf _{y \in Q}\left(\inf _{z \in Q} \delta^{-1}(z)\right)\left(\nu \mathcal{X}_{Q}\right)^{*}(y)\right)\|f \alpha\|_{\frac{n}{\gamma}} \\
& \leq C \sup \left(\inf _{y \in Q} \beta^{-1}(y)\right)\|f \alpha\|_{\frac{n}{7}} .
\end{aligned}
$$

The proof for $M_{9} f$ is parareli to the proof for $M_{3}$.

For $M_{10}^{j}$ we use Hölder's inequality with $r^{\prime}<\frac{n}{\gamma}$ and $r<\frac{n}{n-\gamma}(1+\varepsilon)$ such that $\alpha^{-r} \in A_{1}$ getting

$M_{10}^{j} f(x) \leq \sup \left(\frac{1}{|Q|} \int_{Q}\left\|b(y)-b_{Q}\right\| d y\right)\left(\frac{1}{|Q|} \int_{Q}\left\|a(y)-a_{Q}\right\| d y\right)\left|2^{j} Q\right|^{\gamma / n}$

$\left(\frac{1}{\left|2^{j} Q\right|} \int_{2^{j} Q}\|f(y) \alpha(y)\|^{r^{\prime}} d y\right)^{1 / r^{\prime}}\left(\frac{1}{\left|2^{j} Q\right|} \int_{2^{j} Q} \alpha^{-r}(y) d y\right)^{1 / r}$

$\leq C\|f \alpha\|_{\frac{n}{\gamma}} \sup \left(\frac{1}{|Q|} \int_{Q}\left\|b(y)-b_{Q}\right\| d y\right)\left(\frac{1}{|Q|} \int_{Q}\left\|a(y)-a_{Q}\right\| d y\right)\left(i n f_{y \in 2} Q_{Q} \alpha^{-1}(y)\right)$

$\leq C\|f \alpha\|_{\frac{n}{\gamma}} \sup \left(\frac{1}{|Q|} \int_{Q}\left\|b(y)-b_{Q}\right\| d y\right)\left(\frac{1}{|Q|} \int_{Q}\left\|a(y)-a_{Q}\right\| \alpha^{-1}(y) d y\right)$. 
Now applying Remark (2.11) and Lemma (4.41) twice we obtain the desired result for $M_{10}^{j}$. We don't give the proof for $M_{11}^{j}$ which is a mixture of the proofs for $M_{10}^{j}$ and for $M_{3}$. Analogously the proof of $M_{12}^{j}$ is a mixture of the proofs for $M_{7}$ and $M_{10}^{j}$.

Since $\delta^{-1} \in A_{1}$ we have by Lemma (4.41)

$$
\begin{aligned}
M_{13} f(x) & \leq C\|f \delta\|_{\infty} \sup \left(\frac{1}{|Q|} \int_{Q}\left\|b(y)-b_{Q}\right\| d y\right)\left(\inf _{y \in Q} \delta^{-1}(y)\right) \\
& \leq C\|f \delta\|_{\infty} \cdot \beta^{-1}(x)
\end{aligned}
$$

Finally, if $u=\frac{2 n}{n+\gamma}$, then by Hölder's inequality and lemma (4.43), we have,

$$
\begin{aligned}
& M_{14}^{u} f(x) \leq \sup \left(\frac{1}{|Q|} \int_{Q}\left\|b(y)-b_{Q}\right\| d y\right)\|f \alpha\|_{n / \gamma} \\
& \left(\frac{1}{|Q|} \int_{Q} \alpha^{-n /(n-\gamma)}(y) d y\right)^{(n-\gamma) / 2 n}\left(\frac{1}{|Q|} \int_{Q} \beta^{-u / 2}(y) d y\right)^{1 / u} \\
& \leq \sup \left(\frac{1}{|Q|} \int_{Q}\left\|b(y)-b_{Q}\right\| \alpha^{-1 / 2}(y) d y\right)\|f \alpha\|_{n / \gamma}\left(\frac{1}{|Q|} \int_{Q} \beta^{-u / 2}(y) d y\right)^{1 / u} .
\end{aligned}
$$

Then, applying lemma (4.41) to $\nu=\alpha^{1 / 2} \beta^{-1 / 2}$, we have,

$$
M_{14}^{u} f(x) \leq \sup \beta^{-1 / 2}(x)\|f \alpha\|_{n / \gamma}\left(\frac{1}{|Q|} \int_{Q} \beta^{-u / 2}(y) d y\right)^{1 / u} .
$$

Since $\frac{u}{2}<\frac{n}{n-\gamma}$ then $\beta^{-u / 2} \in A_{1}$ and then we get the desired result.

Proof of Proposition (4.33):

We shall prove (4.34) and (4.37), the other cases can be proved analogously.

Let $Q$ be a cube in $\mathbf{R}^{n}$ with center ar $x_{0}$. Given a function $f$ with compact support, we define

$$
f_{1}(x)=f(x) \mathcal{X}_{2 Q}(x), \quad f_{2}(x)=f(x)-f_{1}(x) .
$$

Let

$$
c_{Q}=T\left(\left(b_{Q}-b\right) f_{2}\right)\left(x_{Q}\right) .
$$

Then if $x \in Q$, we have

$$
\begin{aligned}
C_{b} f(x)= & \tilde{b}(x) T f(x)-T(b f)(x)=\left(\tilde{b}(x)-\tilde{b}_{Q}\right) T f(x) \\
& +T\left(b_{Q} f\right)(x)-T(b f)(x)=\left(\tilde{b}(x)-\tilde{b}_{Q}\right) T f(x) \\
& +T\left(\left(b_{Q}-b\right) f\right)(x) .
\end{aligned}
$$


Therefore, for $x \in Q$, we have

$$
\begin{aligned}
\left\|C_{b} f(x)-c_{Q}\right\|_{F} \leq & \left\|\left(\tilde{b}(x)-\tilde{b}_{Q}\right) T f(x)\right\|_{F} \\
& +\left\|T\left(\left(b_{Q}-b\right) f_{1}\right)(x)\right\|_{F} \\
& +\left\|T\left(\left(b_{Q}-b\right) f_{2}\right)(x)-T\left(\left(b_{Q}-b\right) f_{2}\right)\left(x_{0}\right)\right\|_{F} \\
= & \sigma_{1}(x)+\sigma_{2}(x)+\sigma_{3}(x) .
\end{aligned}
$$

We shall estimate $\left(C_{b} f\right)^{\#}\left(x_{0}\right)$ in terms of the $\sigma_{1}(x)$. Obviously

$$
\frac{1}{|Q|} \int_{Q} \sigma_{1}(x) d x \leq M_{1}(T f)\left(x_{0}\right) \text {. }
$$

Now, for $\sigma_{2}(x)$ choose $r$ such that $\frac{1}{s}-\frac{1}{r^{\prime}}=\frac{\gamma}{n}$ and $s<(1+\varepsilon)$. Then using the boundedness properties of $T$, we have,

$$
\begin{aligned}
& \frac{1}{|Q|} \int_{Q} \sigma_{2}(x) d x \leq\left(\frac{1}{|Q|} \int_{Q}\left\|T\left(\left(b-b_{Q}\right) f_{1}\right)(x)\right\|^{r^{\prime}} d x\right)^{1 / r^{\prime}} \\
& \leq C\left(\frac{1}{|Q|} \int_{Q}\left\|\left(b(x)-b_{Q}\right) f(x)\right\|^{s} d x\right)^{1 / s}|Q|^{\gamma / n} \leq C M_{2}^{s} f\left(x_{0}\right) .
\end{aligned}
$$

On the other hand, by using hypotheses (K.1) and (K.2), we have,

$$
\begin{aligned}
\sigma_{3}(x) & \leq \int\left\|b(y)-b_{Q}\right\|\left\|f_{2}(y)\right\|\left\|K(x, y)-K\left(x_{0}, y\right)\right\| d y \\
& \leq C \sum_{j=1}^{\infty} \frac{|Q|^{1 / n}}{\left|2^{j} Q\right|^{\frac{1}{n}+1-\frac{\gamma}{n}}} \int_{2^{j+1} Q \mathbf{2}^{i} Q}\left\|b(y)-b_{Q}\right\|\|f(y)\| d y \\
& \leq C^{t} \sum_{j=1}^{\infty} \frac{1}{2^{j}}\left\{\frac{1}{\left|2^{j} Q\right|^{1-\gamma / n}} \int_{2^{i} Q}\left\|b(y)-b_{2 i Q}\right\|\|f(y)\| d y\right. \\
& \left.+\frac{1}{\left|2^{j} Q\right|^{1-\gamma / n}} \int_{2^{j} Q}\left\|b_{2 ; Q}-b_{Q}\right\|\|f(y)\| d y\right\} \\
& \leq C \sum_{j} \frac{1}{2^{j}}\left\{M_{2} f\left(x_{0}\right)+\left\|b_{2^{i} Q}-b_{Q}\right\| \frac{1}{\left|2^{j} Q\right|^{1-\gamma / n}} \int_{2^{j} Q}\|f(y)\| d y\right\} .
\end{aligned}
$$

Using (4.38), we get

$$
\begin{aligned}
& \sigma_{3}(x) \leq \\
& C \sum_{j} \frac{1}{2^{j}}\left\{M_{2} f\left(x_{0}\right)+j\left(\inf _{y \in 2^{j} Q}\left(\nu \mathcal{X}_{2}\right)_{Q}^{*}(y)\right)\left(\frac{1}{\left|2^{j} Q\right|^{1-\gamma / n}} \int_{2^{j} Q}\|f(y)\| d y\right)\right\} \\
& \leq C \sum_{j} \frac{1}{2^{j}}\left\{M_{2} f\left(x_{0}\right)+j M_{3} f\left(x_{0}\right)\right\} .
\end{aligned}
$$


This finishes the proof of $(4.34)$.

In order to prove (4.37), given a cube $Q$ and a positive compactly supported function $f$, we decompose $f$ into $f_{1}$ and $f_{2}$ as before and we consider

$$
w_{Q}=\int\left|a_{Q}-a(y) \| b_{Q}-b(y)\right| W\left(x_{0}, y\right) f_{2}(y) d y .
$$

We observe that $w_{Q}$ is finite since $b_{Q}-b$ and $a_{Q}-a$ belongs to $L^{2}(Q)$. If $x \in Q$ and $|\cdot|$ is the absolute value in $F$, standard computations give

$$
\begin{aligned}
\left|V_{a_{b} b}^{+} f(x)-w_{Q}\right| \leq & \int \mid(a(x)-a(y))(b(x)-b(y)) W(x, y) f(y) \\
& -\left(a_{Q}-a(y)\right)\left(b_{Q}-b(y)\right) W\left(x_{0}, y\right) f_{2}(y) \mid d y \\
\leq & \left|a(x)-a_{Q}\right| \int|b(x)-b(y)| W(x, y) f(y) d y \\
& +\left|b(x)-b_{Q}\right| \int\left|a_{Q}-a(y)\right| W(x, y) f_{1}(y) d y \\
& +\left|b(x)-b_{Q}\right| \int\left|a_{Q}-a(y)\right| W(x, y) f_{2}(y) d y \\
& +\int\left|a_{Q}-a(y)\right|\left|b_{Q}-b(y)\right| W(x, y) f_{1}(y) d y \\
& +\int\left|a_{Q}-a(y) \| b_{Q}-b(y)\right| W(x, y)-W\left(x_{0}, y\right) \mid f_{2}(y) d y \\
= & \lambda_{1}(x)+\lambda_{2}(x)+\lambda_{3}(x)+\lambda_{4}(x)+\lambda_{5}(x) .
\end{aligned}
$$

For $\lambda_{3}$, and since $a_{Q}=\frac{1}{|Q|} f_{Q} a(z) d z$ we have

$$
\lambda_{3}(x)=\left|b(x)-b_{Q}\right| \int\left|\frac{1}{Q} \int_{Q}(a(z)-a(y)) W(x, y) f_{2}(y) d z\right| d y .
$$

Then,

$$
\begin{aligned}
& \lambda_{3}(x) \leq\left|b(x)-b_{Q}\right| \frac{1}{|Q|} \int_{Q} \int|a(z)-a(y)| W(x, y)-W(z, y) \mid f_{2}(y) d y d z \\
& \quad+\left|b(x)-b_{Q}\right|\left(\frac{1}{|Q|} \int_{Q} V_{a}^{+}(f)(z) d z\right)+\left|b(x)-b_{Q}\right|\left(\frac{1}{|Q|} \int_{Q} V_{a}^{+}\left(f_{1}\right)(z) d z\right) \\
& =\lambda_{3,1}(x)+\lambda_{3,2}(x)+\lambda_{3,3}(x) .
\end{aligned}
$$

It is clear that

$$
\frac{1}{|Q|} \int_{Q}\left\|\lambda_{1}(x)\right\| d x \leq M_{4}\left(V_{b}^{+} f\right)\left(x_{0}\right)
$$


232

C. Segovia, J.L. Torres

Choose $u=\frac{2 n}{n+\gamma}$, then $\frac{1}{u}-\frac{1}{u^{\prime}}=\frac{\gamma}{n}$. Therefore by the hypothesis on $V$, we have,

$$
\begin{aligned}
& \frac{I}{|Q|} \int_{Q}\left\|\lambda_{2}(x)\right\| d x \\
& \quad \leq\left(\frac{1}{|Q|} \int_{Q}\left|b(x)-b_{Q}\right|^{u} d x\right)^{1 / u}\left(\frac{1}{|Q|} \int\left\|V\left(\left|a_{Q}-a\right| f_{1}\right)(x)\right\|^{u^{\prime}} d x\right)^{1 / u^{\prime}} \\
& \leq C\left(\frac{1}{|Q|} \int_{Q}\left|b(x)-b_{Q}\right|^{u} d x\right)^{1 / u}|Q|^{\gamma / n-1 / u}\left(\int_{Q}\left(\left|a_{Q}-a(x)\right| f(x)\right)^{u} d x\right)^{1 / u} \\
& \leq C M_{5}^{u} f\left(x_{0}\right) .
\end{aligned}
$$

In order to handle $\lambda_{3,1}(x)$ we observe that

$$
\begin{aligned}
& \frac{1}{|Q|} \int_{Q}\left(|a(z)-a(y)||W(x, y)-W(z, y)| f_{2}(y) d y\right) d z \\
& \leq \frac{1}{|Q|} \int_{Q}\left(\sum_{j} \frac{1}{2^{j}\left|2^{j} Q\right|^{1-\gamma / n}} \int_{2^{\prime} Q}|a(z)-a(y)| f(y) d y\right) d z \\
& \leq \sum_{j} \frac{\left|2^{j} Q\right|}{2^{j}} \frac{1}{|Q|} \int_{Q}\left(\frac{1}{\left|2^{j} Q\right|} \int_{2^{i} Q}\left|a(z)-a_{Q}+a_{Q}-a_{2^{j} Q}+a_{2^{j} Q}-a(y)\right| f(y)\right) d z \\
& \leq \sum_{j} \frac{\left|2^{j} Q\right|^{\gamma / n}}{2^{j}} \frac{1}{|Q|} \int_{2^{j},} \frac{1}{\left|2^{j} Q\right|} \int_{2^{j} Q}\left|a(z)-a_{Q}\right| f(y) d y d z \\
& +\sum_{j} \frac{\left|2^{j} Q\right|^{\gamma / n}}{2^{j}} \frac{1}{|Q|} \int_{Q} \frac{1}{\left|2^{j} Q\right|} \int_{2^{i} Q}\left|a_{Q}-a_{2^{j}}\right| f(y) d y d z \\
& +\sum_{j} \frac{\left|2^{j} Q\right|^{\gamma / n}}{2^{j}} \frac{1}{|Q|} \int_{Q} \frac{1}{\left|2^{j} Q\right|} \int_{2 ; Q}\left|a_{2 j Q}-a(y)\right| f(y) d y d z \text {. }
\end{aligned}
$$

By Lemma (4.38) this is less than or equal to

$$
\begin{aligned}
& \sum_{j} \frac{\left|2^{j} Q\right|^{\gamma / n}}{2^{j}}\left(\frac{1}{|Q|} \int_{Q}\left|a(z)-a_{Q}\right| d z\right)\left(\frac{1}{\left|2^{j} Q\right|} \int_{2^{j} Q} f(y) d y\right) \\
& +C \sum_{j}\left|2^{j} Q\right|^{\gamma / n} \frac{j}{2^{j}}\left(\inf _{y \in 2^{j} Q}\left(\nu \mathcal{X}_{Q}\right)^{*}(y)\right)\left(\frac{1}{\left|2^{j} Q\right|} \int_{2^{j} Q} f(y) d y\right) \\
& +\sum_{j} \frac{\left|2^{j} Q\right|}{2^{j}}\left(\frac{1}{\left|2^{j} Q\right|} \int_{2^{j} Q}\left|a_{2^{j} Q}-a(y)\right| f(y) d y\right) .
\end{aligned}
$$


Therefore,

$$
\begin{gathered}
\sup \frac{1}{|Q|} \int_{Q}\left\|\lambda_{3,1}(x)\right\|_{F} d x \leq C \sum_{j}\left\{2^{-j} M_{10}^{j} f\left(x_{\theta}\right)\right. \\
\left.+j 2^{-j} M_{11}^{j} f\left(x_{\theta}\right)+2^{-j} M_{12}^{j} f\left(x_{0}\right)\right\} .
\end{gathered}
$$

It is clear that

$$
\frac{1}{|Q|} \int_{Q}\left\|\lambda_{3,2}(x)\right\| d x \leq C M_{13}\left(V_{a}^{+} f\right)\left(x_{0}\right)
$$

On the other hand if $u=\frac{2 \pi}{n+\gamma}$, we have $\frac{1}{u}-\frac{1}{u^{\prime}}=\frac{\gamma}{n}$, then by lemma (4.47) and Theorem (2.7) for the case $\alpha^{1 / 2} \beta^{-1 / 2}=\nu$, we have,

$$
\begin{aligned}
& \frac{1}{|Q|} \int_{Q}\left\|\lambda_{33}(x)\right\| d x \leq\left(\frac{1}{|Q|} \int_{Q}\left|b(x)-b_{Q}\right| d x\right) \\
& \left(\frac{1}{|Q|} \int_{Q}\left\|V_{a}^{+} f_{1}(z)\right\|^{u^{\prime}} \beta^{u^{\prime} / 2}(z) d z\right)^{1 / u^{\prime}}\left(\frac{1}{|Q|} \int_{Q} \beta^{-u / 2}(z) d z\right)^{1 / u} \\
& \leq\left(\frac{1}{|Q|} \int_{Q}\left|b(x)-b_{Q}\right| d x\right)|Q|^{\gamma / n}\left(\frac{1}{|Q|} \int_{Q} f(z)^{u} \alpha^{u / 2}(z) d z\right)^{1 / u} \\
& \left(\frac{1}{|Q|} \int_{Q} \beta^{-u / 2}(z) d z\right)^{1 / u} \leq C M_{14}^{u} f\left(x_{0}\right) .
\end{aligned}
$$

We handle $\lambda_{4}(x)$ as follows. Choose $\frac{1}{s}-\frac{1}{r^{\prime}}=\frac{\gamma}{n}$ and $1 \leq s<(1+\varepsilon)$, then by the hypotheses on $V$, we have,

$$
\begin{aligned}
& \frac{1}{|Q|} \int_{Q}\left\|\lambda_{4}(x)\right\| d x \leq\left(\frac{1}{|Q|} \int_{Q}\left\|V\left(\left|a_{Q}-a \| b_{Q}-b\right| f_{1}\right)(x)\right\|^{r^{\prime}} d x\right)^{1 / r^{\prime}} \\
& \leq C|Q|^{\gamma / n}\left(\frac{1}{|Q|} \int_{Q}\left(\left|a_{Q}-a(x) \| b_{Q}-b(x)\right| f(x)\right)^{s} d x\right)^{1 / s} \leq C M_{6}^{s} f\left(x_{0}\right) .
\end{aligned}
$$

Finally we observe that by (W.3), we have,

$$
\begin{aligned}
& \left\|\lambda_{5}(x)\right\| \leq C \sum_{j} \frac{\left|2^{j} Q\right|^{\gamma / n}}{2^{j}\left|2^{j} Q\right|} \int_{2^{j} Q}\left|a_{Q}-a(y) \| b_{Q}-b(y)\right| f(y) d y, \quad \text { and } \\
& \int_{2 i Q}\left|a_{Q}-a(y) \| b_{Q}-b(y)\right| f(y) d y \\
& \leq \int_{2 j Q}\left|a_{2 j} j_{Q}-a(y)\right|\left|b_{2} j_{Q}-b(y)\right| f(y) d y+\left|a_{Q}-a_{2 j}{ }^{2}\right| \int_{2^{j} Q}\left|b_{2 i} Q-b(y)\right| f(y) d y \\
& +\left|b_{Q}-b_{2 j Q}\right| \int_{2 i_{Q}}\left|a_{2 j} j_{Q}-a(y)\right| f(y) d y+\left|b_{Q}-b_{2 j}{ }_{Q}\right|\left|a_{Q}-a_{2} i_{Q}\right| \int_{2 i Q} f(y) d y .
\end{aligned}
$$


By lemma (4.38), this is less than

$$
\begin{aligned}
& \int_{2^{i} Q}\left|a_{2^{i} Q}-a(y) \| b_{2^{j} Q}-b(y)\right| f(y) d y \\
& +j\left(\inf _{y \in 2^{i} Q}\left(\nu \mathcal{X}_{2 j Q}\right)^{*}(y)\right) \int_{2 ; Q}\left|b_{2 j Q}-b(y)\right| f(y) d y \\
& +j\left(\inf _{y \in 2^{i} Q}\left(\nu \mathcal{X}_{2 i Q}\right)^{*}(y)\right)\left(\int_{2^{i} Q}\left|a_{2 i Q}-a(y)\right| f(y) d y\right) \\
& +j^{2}\left(\inf _{y \in 2^{j} Q}\left(\nu \mathcal{X}_{2 j Q}\right)^{*}(y)\right)^{2}\left(\int_{2 ; Q} f(y) d y\right) \text {. }
\end{aligned}
$$

Therefore

$$
\begin{aligned}
\left\|\lambda_{5}(x)\right\| \leq & C\left\{\sum _ { j = 1 } ^ { \infty } \left(2^{-j} M_{6}^{1} f\left(x_{0}\right)+j 2^{-j} M_{7} f\left(x_{0}\right)\right.\right. \\
& \left.+j 2^{-j} M_{8} f\left(x_{0}\right)+j^{2} 2^{-j} M_{9} f\left(x_{0}\right)\right\} .
\end{aligned}
$$

Then, we have,

$$
\left\|\lambda_{5}(x)\right\| \leq C\left\{M_{6}^{1} f\left(x_{0}\right)+M_{7} f\left(x_{0}\right)+M_{8} f\left(x_{0}\right)+M_{9} f\left(x_{0}\right)\right\},
$$

ending the proof of (4.37).

\section{References}

1. S. BLOOM, A commutator theorem and weighted BMO, Trans. Amer. Math. Soc. 292 (1985), 103-22.

2. D.L. BURKIOLDER, A geometric condition that implies the existence of certain singular integrals of Banach-space-valued function, Conference on Harmonic Analysis in Honor of Antony Zygmund, University of Chicago, 1981, Wadsworth International Group, Belmont, California.

3. S. Chanlllo, A Note of Commutators, Indiana Univ. Math. J. 31 (1982), $7-16$.

4. R. A. HuNT, On the convergence of Fourier Series, orthogonal expansions and their continuous analogues, Proc. Conf. Edwardsville (1967), Southern Illinois Univ. Press, Carbondale, Ill., 1968, 235-255.

5. J.L. Rubio de Francia, F.J. Ruiz and J.L. Torrea, CalderónZygmund theory for operator-valued kernels, Adv. in Math. 62 (1986), $7-48$.

6. F.J. RUIZ AND J.L. TORREA, Weighted and vector valued inequalities for Potential Operators, Trens. Amer. Math. Soc. 295 (1986), 213-232. 
7. C. SEGOVIA AND J.L. TORREA, Extrapolation for pairs of related weights, Volume in Honor of M. Cotlar, Marcel Dekker (1989).

8. C. Segovia and J.L. Torrea, Vector-valued commutators and applications, Indiana Univ. Math. J. 38 (1989), 959-971.

9. J.O. STHOMBERG AND R.L. WHEEDEN, Fractional integrals on weighted $H^{p}$ and $L^{p}$ spaces, Trans. Amer. Math. Soc. 287 (1987), 293-321.

10. E. Harboure, R.A. Macías and C. Segovia, Extrapolation results for classes of weights, Amer. J. Math. 110 (1988), 383-397.

Carlos Segovia: IAM, CONICET

Universidad de Buenos Aires (FCE y $N$ )

ARGENTINA

José L. Torrea: Departamento de Matemáticas

Universidad Autonoma de Madrid

28049 - Madrid

SPAIN 